\title{
Blood Pressure May Be Associated with Arterial Collateralization in Anterior Circulation Ischemic Stroke before Acute Reperfusion Therapy
}

\author{
Beisi Jiang, ${ }^{\mathrm{a}, \mathrm{b}}$ Leonid Churilov, ${ }^{\mathrm{c}, \mathrm{d}}$ Lasheta Kanesan, ${ }^{\mathrm{b}}$ Richard Dowling, ${ }^{\mathrm{e}}$ Peter Mitchell, ${ }^{\mathrm{e}}$ Qiang Dong, ${ }^{\mathrm{a}}$ \\ Stephen Davis, ${ }^{\mathrm{b}}$ Bernard Yan ${ }^{\mathrm{b}, \mathrm{e}}$ \\ ${ }^{a}$ Department of Neurology, State Key Laboratory of Medical Neurobiology, Huashan Hospital, Fudan University, Shanghai, China \\ bDepartment of Medicine and Neurology, Melbourne Brain Centre at the Royal Melbourne Hospital, University of Melbourne, Parkville, Australia \\ 'Statistics and Decision Analysis, Florey Institute of Neuroscience and Mental Health, Parkville, Australia \\ 'School of Mathematics and Geospatial Science, RMIT University, Melbourne, Australia \\ eDepartment of Radiology, Neurointervention Service, Royal Melbourne Hospital, University of Melbourne, Parkville, Australia
}

Background and Purpose Leptomeningeal collaterals maintain arterial perfusion in acute arterial occlusion but may fluctuate subject to arterial blood pressure (ABP). We aim to investigate the relationship between $\mathrm{ABP}$ and collaterals as assessed by computer tomography (CT) perfusion in acute ischemic stroke.

Methods We retrospectively analyzed acute anterior circulation ischemic stroke patients with CT perfusion from 2009 to 2014. Collateral status using relative filling time delay (rFTD) determined by time delay of collateral-derived contrast opacification within the Sylvian fissure, from 0 seconds to unlimited count. The data were analyzed by zero-inflated negative binomial regression model including an appropriate interaction examining in the model in terms of occlusion location and onset-to-CT time (OCT).

Results Two hundred and seventy patients were included. We found that increment of $10 \mathrm{~mm} \mathrm{Hg}$ in $\mathrm{BP}$, the odds that a patient would have $\mathrm{FFD}$ equal to 0 seconds increased by $27.9 \%$ in systolic BP (SBP) $(P=0.001)$, by $73.9 \%$ in diastolic BP (DBP) $(P<0.001)$ and by $68.5 \%$ in mean BP (MBP) $(P<0.001)$. For patients with $r F T$ not necessarily equal to 0 seconds, every $10 \mathrm{~mm} \mathrm{Hg}$ increase in $\mathrm{BP}$, there was a 7\% decrease in expected count of seconds for $\mathrm{rFT}$ in SBP $(P=0.002), 10 \%$ decrease for rFTD in DBP and 11\% decrease for rFTD in MBP. The arterial occlusion location and OCT showed no significant interaction in the BP-rFT relationship ( $P>0.05)$.

Conclusions In acute ischemic stroke, higher ABP is possibly associated with improved leptomeningeal collaterals as identified by decreased rFTD.

Keywords Acute ischemic stroke; Blood pressure; Collaterals; Computed tomography perfusion; Recanalization

\author{
Correspondence: Bernard Yan \\ Department of Medicine and \\ Neurology, Melbourne Brain Centre at \\ the Royal Melbourne Hospital; \\ Department of Radiology, \\ Neurointervention Service, Royal \\ Melbourne Hospital, University of \\ Melbourne, 300 Grattan Street, \\ Parkville, Victoria 3050, Australia \\ Tel: +61-3-9349-2477 \\ Fax: +61-3-9349-4489 \\ E-mail: Bernard.yan@mh.org.au \\ Received: November 14, 2016 \\ Revised: January 6, 2017 \\ Accepted: January 22, 2017 \\ The authors have no financial conflicts \\ of interest.
}

\section{Introduction}

Leptomeningeal collaterals provide an important pathway in the maintenance of blood flow to brain regions distal to an arterial occlusion, ${ }^{1-5}$ critically influencing clinical outcomes, ${ }^{6-8}$ especially during the ultra-early ischemic phase before recanali- 
zation treatment. ${ }^{9}$ In addition, autoregulation of collaterals varies, leading to dependence of at-risk tissue on systolic mean arterial pressure. ${ }^{10}$ The corollary is that increase in arterial blood pressure may improve arterial perfusion in acute ischemic stroke, leading to minimization of final infarct volume in animal model. ${ }^{11}$ Furthermore, raised arterial blood pressure may reduce hypoperfusion in acute ischemic stroke. ${ }^{12,13}$ Of note, a U-shaped relationship between blood pressure on hospital admission during the acute phase of ischemic stroke and mortality has been described, ${ }^{14-16}$ suggesting that undue hypotension leads to worse outcome, likely explained by underperfusion. However, the positive influence of higher mean arterial pressure on leptomeningeal collateral flow remains unsubstantiated.

In this study, we aim to investigate the impact of blood pressure on arterial collaterals. According to our previous research findings, relative filling time delay (rFTD), ${ }^{17}$ a surrogate marker for collaterals, utilizing computer tomography perfusion source imaging (CTP-SI) to assess leptomeningeal collaterals indirectly (time delay [in seconds] of collateral-derived contrast opacification within the Sylvian fissure), has suggested a significant association with the size of infarct volume and clinical outcomes. We hypothesize that in hyperacute and pre-reperfusion-treatment ischemic stroke patients, increase in arterial blood pressure is associated with decrease in rFTD, indicating improved leptomeningeal collaterals.

\section{Methods}

\section{Study design and patient cohort}

This was a single-center retrospective cohort study involving patients with acute anterior circulation ischemic stroke who received intravenous altepase within 4.5 hours after stroke and/or intra-arterial therapy within 6 hours at the Royal Melbourne Hospital during the period from January 2009 to December 2014. This study was approved by Melbourne Health Human Research Ethics Committee.

Patients were eligible for inclusion if they underwent computer tomography (CT) perfusion before any recanalization treatment and had confirmed records of systemic blood pressure before imaging. While those who had stroke-like symptoms without any reliable evidences on neuroimaging (including non-contrast CT, CT perfusion and magnetic resonance imaging diffusion) to support the diagnosis were excluded.

\section{Clinical information}

Clinical parameters, identified through computerized databases, were collected. The following baseline parameters were col- lected: (1) demographics (age, gender); (2) vascular risk factors including: hypertension, diabetes mellitus, history of smoking, hyperlipidemia, ischemic heart disease, atrial fibrillation, previous stroke and transient ischemic attack (TIA); (3) stroke severity assessed by National Institutes of Health Stroke Scale (NIHSS). Systemic blood pressure acquired before CT perfusion, including systolic blood pressure (SBP), diastolic blood pressure (DBP) and mean blood pressure (MBP) which was calculated by the following formula $\mathrm{MBP}=[\mathrm{SBP}+2 \times \mathrm{DBP}] / 3$. The time of onset to $\mathrm{CT}$ perfusion performing was identified as the period between the time point of symptoms onset or when the patient was last seen neurologically well and the time of CT perfusion by the minute.

\section{Imaging parameters}

CT perfusion data were obtained from pictures archiving and communication system in Royal Melbourne Hospital on a range of hardware platforms using protocols based on current consensus recommendations. ${ }^{18,19}$ Before 2013, images were acquired by 16 -section Somatom CT (Siemens, Erlangen, Germany; two separate CT perfusion slabs with 24-mm thick). During 2013 till the end of 2014, Flash 128 Slice CT was available routinely (Siemens, Definition, Germany; two separate CT perfusion slabs with $10-\mathrm{mm}$ thick). lodinated contrast (40-mL iohexol $350 \mathrm{mgl} / \mathrm{mL}$, Omnipaque 350; GE Healthcare, Milwaukee, WI, USA) was injected at $8 \mathrm{~mL} / \mathrm{s}$, and 40 images were acquired per second (total acquisition time, 44 seconds).

\section{Imaging analysis}

CT perfusion images for each patient were reviewed by two neurologists and one research fellow trained in neuroimaing (B.J., B.Y., L.K.) with consensus opinion reached on collateral supply. The status of the leptomeningeal collaterals was evaluated using rFTD identified by unprocessed CTP-SI DICOM images between the normal middle cerebral artery Sylvian branches and those in the affected hemisphere. It was defined as the time difference (in seconds) between the first appearances of contrast in each Sylvian fissure (Figure 1). All reviewers were blinded to clinical outcome during the agreement assessment, and measured by inter-reviewer Kappa in statistical analysis.

\section{Statistical analysis}

Statistical analysis was performed using STATA, version 13.1 (StataCorp, College Station, TX, USA). $P<0.05$ was considered to indicate statistical significance. We compared the differences of characteristics at baseline between included and excluded cohorts using the Fisher exact test for categorical variables and 

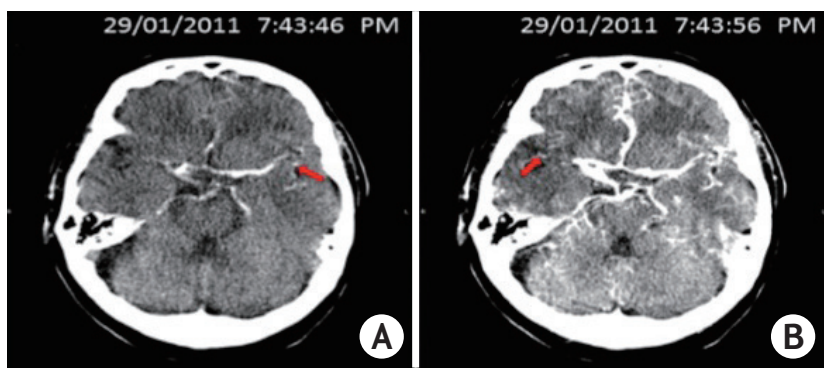

Figure 1. This picture showed a typical case of relative filling time delay (rFTD): a 71-year-old male patient presenting with left hemiplegia and dysphasia arrived hospital and underwent computer tomography examination 81 minutes after onset. Computer tomography angiography indicated the occluded right internal carotid artery and M1 segment of the right middle cerebral artery. Computer tomography perfusion source imaging (CTP-SI) illustrated that contrast signal (red arrow) firstly appeared in the normal side's Sylvian fissure in 7:43:46 PM (A), whereas showed up in the occlusion side's Sylvain fissure in 7:43:56 PM (B), therefore, the time gap of $10 \mathrm{~s}$ between bilateral contrast appearances in Sylvian fissure was identified as an rFTD.

the Wilcoxon-Mann-Whitney test for continuous variables.

Association between rFTD (in seconds) and BP (involving SBP, DBP, and MBP) was estimated using a zero-inflated negative binomial regression model (ZINB). ${ }^{20}$ ZINB model is appropriate in situations where zero outcomes are overrepresented (in this study it means 0 seconds rFTD). Such model assumes that there exist two latent groups: an "always zero" group that has the count outcome of 0 seconds rFTD with certainty, and a "not always zero" group that may have a 0-second or a more-than0 -second in rFTD. The model estimates two sets of coefficients: the coefficients estimating change in the odds of belonging to "always zero" group compared to "not always zero" group by $10 \mathrm{~mm} \mathrm{Hg}$ of blood pressure (holding all other variables constant), and, for "not always zero" group, the coefficients describing an expected change in the count of rFTD seconds by $10 \mathrm{~mm} \mathrm{Hg}$ of blood pressure (holding all other variables constant). Specific regression models were chosen on the combination of standard fit criteria and tests as implemented in SPost suit of command in STATA. ${ }^{21}$

The interaction of different arterial occlusion locations (e.g. middle cerebral artery [MCA] segment 1 or 2 , internal carotid artery [ICA], etc.) and OCT in rFTD-BP association were examined by including appropriate interaction terms in the model.

\section{Results}

Between January 2009 and December 2014, 451 patients with definite diagnosis of acute anterior circulation ischemic stroke presented to our institution within 4.5 hours of stroke-symptom onset for intravenous tissue plasminogen activator and/or

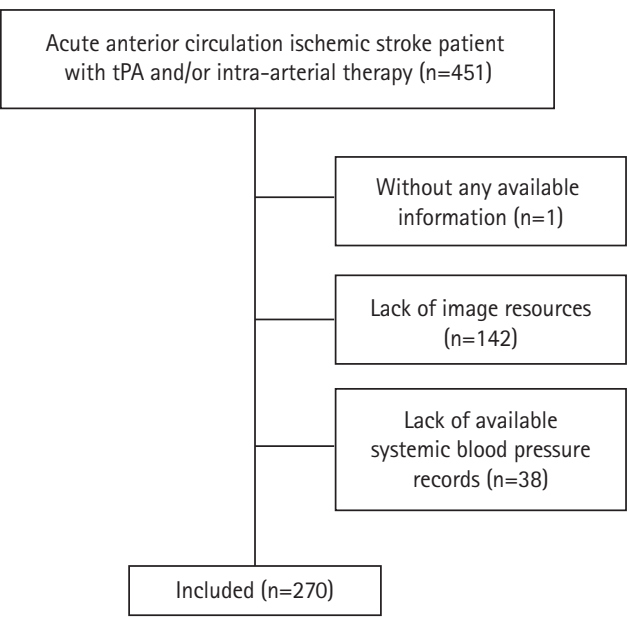

Figure 2. Flow chart showing selection of eligible patients. tPA, tissue plasminogen activator.

within 6 hours for retrieval therapy. The following patients were excluded: 1 patient lacked clinical information (e.g. involving medical history, imaging resources, onset blood pressure), 142 patients lacked imaging resources, 38 patients lacked blood pressure records (Figure 2).

\section{Baseline characteristics}

Two hundred and seventy patients were included into this study with completed CTP imaging and available blood pressure records. When compared with the excluded cohort, there were no significant differences in baseline characters between these two groups except NIHSS with a significant higher median score in included patients. The median age of patients was 74 yrs (q25-75: 64-80), with 44.81\% (121/270) being women. Regarding vascular risk factors, hypertension was found in 65.93\% (178/270); diabetes mellitus, in 26.67\% (72/270); hyperlipidemia, in 40.69\% (106/270); previous ischemic heart disease, in 18.89\% (51/270), atrial fibrillation, in 37.04\% (100/270); former and current smoker combined, in 25.93\% (70/270); previous stroke or TIA history, in 12.59\% (34/270) and 6.3\% (17/270); and median NIHSS was 16 (q25-75: 10-20) (Table 1).

\section{Relationship between arterial BP and rFTD}

The variables of blood pressure and collateral circulation were analyzed. Overall, the MBP of included cohort ranged from $63.3 \mathrm{~mm} \mathrm{Hg}$ to $144.7 \mathrm{~mm} \mathrm{Hg}$, with a mean of $102.4 \mathrm{~mm} \mathrm{Hg}$, and the mean SBP was $148.5 \mathrm{~mm} \mathrm{Hg}(\min =90.0, \max =227.0)$ as well as DBP with mean level $79.4 \mathrm{~mm} \mathrm{Hg}(\mathrm{min}=38.0$, $\max =121.0)$. rFTD of this group was from 0 second up to a maximum of 17 seconds, medium time was 3 seconds (q25-75: 
Table 1. Baseline characteristics for all patients and categorized by include and exclude criterion

\begin{tabular}{lcccc}
\hline Baseline parameter & All patients $(n=451)$ & Included $(\mathrm{n}=270)^{*}$ & Excluded $(\mathrm{n}=181)^{\dagger}$ & $P($ Include vs. Exclude) \\
\hline Demographics & & & & \\
Age (years) & $73.5(62-80)$ & $74(64-80)$ & $73(58-80)$ & 0.478 \\
$\quad$ Gender (female) & $211 / 450(46.9)$ & $121 / 270(44.8)$ & $90 / 180(50.0)$ & 0.280 \\
Clinical history & & & & \\
Hypertension & $289 / 499(64.4)$ & $178 / 270(65.9)$ & $111 / 179(62.0)$ & 0.396 \\
Diabetes mellitus & $118 / 449(26.3)$ & $72 / 270(26.7)$ & $46 / 179(25.7)$ & 0.819 \\
Smoke & $107 / 430(24.9)$ & $70 / 270(25.9)$ & $37 / 160(23.1)$ & 0.516 \\
Hyperlipidemia & $185 / 449(41.2)$ & $106 / 270(39.3)$ & $79 / 179(44.1)$ & 0.304 \\
Ischemic heart disease & $91 / 449(20.3)$ & $51 / 270(18.9)$ & $40 / 179(22.4)$ & 0.372 \\
Atrial fibrillation & $158 / 449(35.2)$ & $100 / 270(37.0)$ & $58 / 179(32.4)$ & 0.314 \\
Previous stroke & $55 / 449(12.3)$ & $34 / 270(12.6)$ & $21 / 179(11.7)$ & 0.785 \\
Previous TIA & $23 / 449(5.1)$ & $17 / 270(6.3)$ & $6 / 179(3.4)$ & 0.166
\end{tabular}

Artery occlusions

ICA

$58 / 270$ (21.5)

MCA-M1

$95 / 270(35.2)$

MCA-M2 and distal

$116 / 270(43.0)$

ACA

$1 / 270(0.3)$

Onset status

NIHSS

$14(8-19)$

$16(10-20)$

13 (7-19)

0.0016

Values are presented as median (IQR) or $\mathrm{n}(\%)$.

$I Q R$, interquartile range (denoted by 25 th and 75 th percentile); TIA, transient ischemic attack; ICA, internal carotid artery; MCA-M1, middle cerebral artery segment 1; MCA-M2, middle cerebral artery segment 2; ACA, anterior cerebral artery; NIHSS, National Institutes of Health Stroke Scale.

*Patients with a pre-treatment blood pressure and undertaken CTP.

+Patients without a pre-treatment blood pressure and/or undertaken CTP.

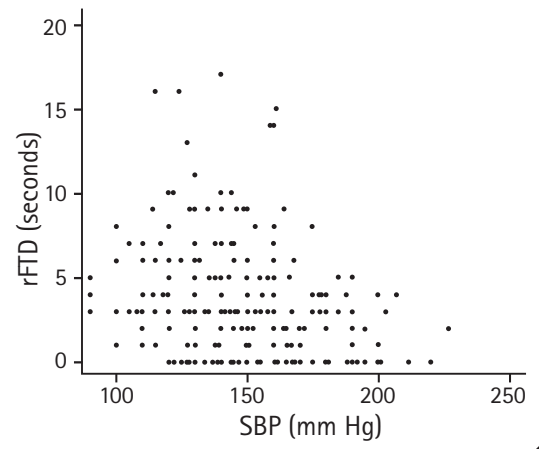

A

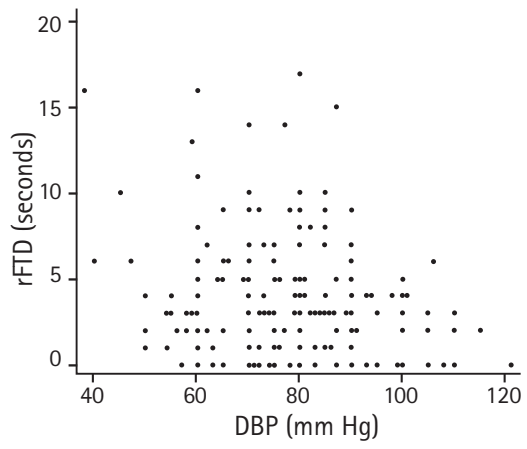

B

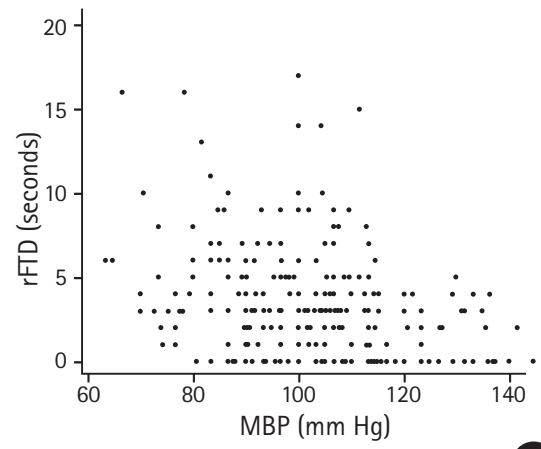

(1)

Figure 3. Scatter-plot for the correlation between rFTD and SBP, DBP, and MBP. rFTD, relative filling time delay; SBP, systolic blood pressure; DBP, diastolic blood pressure; MBP, mean blood pressure.

$0-5 \mathrm{~s})$ with a large proportion of 0 second $(68 / 270,25.2 \%)$. The median OCT of these patients was 96.5mins (q25-75: 71-136 mins). The inter-observer consensus Kappa value is 0.74 .

The relationship between rFTD and ABP was analyzed. The scatter-plot showed that the ABP, including SBP, DBP and MBP, was negatively associated with rFTD (Figure 3). The logistic regression part of the zero-inflated negative binomial regression model which investigated the association between whether or not $\mathrm{rFT}$ was 0 second and BP showed that every increase of $10 \mathrm{~mm} \mathrm{Hg}$ in SBP, the odds that a patient would have rFTD equal to zero would increase by $27.9 \%$ (odds ratio $[O R]=1.279$, 95\% confidence interval [CI]: 1.106-1.479, $P=0.001)$. The similar relationships were for DBP $(\mathrm{OR}=1.739,95 \% \mathrm{Cl}$ : 1.3022.322, $P<0.001)$ and $\mathrm{MBP}(\mathrm{OR}=1.685,95 \% \mathrm{Cl}: 1.309-2.170$, 
Table 2. Zero-inflated negative binomial regression analyses: blood pressure is associated with rFTD

\begin{tabular}{|c|c|c|c|c|}
\hline & \multicolumn{2}{|c|}{$\begin{array}{l}\text { Factor change in expected count of seconds } \\
\text { if rFTD is not } 0 \text { seconds }\end{array}$} & \multicolumn{2}{|c|}{ Factor change in odds of rFTD being 0 seconds } \\
\hline & $\operatorname{IRR}^{*}(95 \% \mathrm{Cl})$ & $P$ & $\mathrm{OR}^{+}(95 \% \mathrm{Cl})$ & $P$ \\
\hline Systolic blood pressure per $10 \mathrm{~mm} \mathrm{Hg}$ & $0.93(0.89-0.98)$ & 0.002 & $1.279(1.106-1.479)$ & 0.001 \\
\hline Mean blood pressure per $10 \mathrm{~mm} \mathrm{Hg}$ & $0.90(0.83-0.97)$ & 0.006 & $1.739(1.302-2.322)$ & $<0.001$ \\
\hline Mean blood pressure per $10 \mathrm{~mm} \mathrm{Hg}$ & $0.89(0.83-0.95)$ & 0.001 & $1.685(1.309-2.170)$ & $<0.001$ \\
\hline
\end{tabular}

rFTD, relative filling time delay; IRR, incidence rate ratio; $\mathrm{Cl}$, confidence interval; $\mathrm{OR}$, odds ratio.

*The factor change in rFTD (expected count of seconds) per $10 \mathrm{~mm} \mathrm{Hg.} \mathrm{IRR>1} \mathrm{indicates} \mathrm{an} \mathrm{increase} \mathrm{of} \mathrm{rFTD} \mathrm{(seconds)} \mathrm{per} \mathrm{increase} \mathrm{of} 10 \mathrm{~mm} \mathrm{Hg}$, while IRR<1 indicates an decrease of rFTD (seconds) per increase of $10 \mathrm{~mm} \mathrm{Hg}$.

'The factor change in odds of having 0 seconds $r F T$ per $10 \mathrm{~mm} \mathrm{Hg}$ increase. $\mathrm{OR}>1$ indicates an increase of odds of 0 seconds $r F T D$ per $10 \mathrm{~mm} \mathrm{Hg}$, while $\mathrm{OR}<1$ indicates an decrease of odds of 0 seconds rFT per $10 \mathrm{~mm} \mathrm{Hg}$.

$P<0.001)$ (Table 2). In other words, higher levels of BP are associated with the higher odds of 0 second rFTD. At the same time, for the patients with rFTD not necessarily equal to zero, we showed that for every $10 \mathrm{~mm} \mathrm{Hg}$ increase in SBP, there was a 7\% decrease in expected count of seconds for rFTD (incidence rate ratio $[\mathrm{IRR}]=0.93,95 \% \mathrm{Cl}$ : $0.89-0.98, P=0.002$ ). The similar relationships were also found in DBP (IRR=0.90,95\% $\mathrm{Cl}: 0.83-0.97, P=0.006)$ and MBP (IRR=0.89, 95\% Cl: $0.83-$ 0.95, $P=0.001$ ) (Table 2). This means that higher levels of $B P$ are associated with shorter rFTD.

Additionally, we undertook a test of interaction of the influence of different arterial occlusion location (e.g. MCA segment 1 or 2, ICA, etc.) and OCT in the association between BP and $r F T$. We found that there was no significant interaction identified in the BP-rFTD association ( $P>0.05$ ).

\section{Discussion}

We showed a significant correlation between rFTD and systemic blood pressure. By ZINB regression analysis, we found that, for every $10 \mathrm{mmHg}$ increase in systolic blood pressure, rFTD decreased by $7 \%$, suggesting improved collateral status.

Previous studies demonstrated inconclusive findings concerning the relationship between arterial blood pressure and collateral circulation. ${ }^{7,8,22}$ Lima et al., ${ }^{7}$ Calleja et al. ${ }_{1}^{6}$ and Liebeskind et al. ${ }^{8}$ did not show significant association between blood pressure, collateral circulation and clinical outcome. Rusanen et al. ${ }^{22}$ found favorable collateral circulation was associated with moderately elevated SBP, especially in the 170-190 mm $\mathrm{Hg}$ range, but not reaching statistical significance. On the other hand, induce hypertension therapy has been mentioned with potential benefit on cerebral perfusion ${ }^{23}$ and antihypertension treatment possibly linked to higher mortality. ${ }^{24}$ In our study, we showed positive relationship between SBP and collaterals and likewise, MBP and DBP were also significantly associated with collaterals. Although we did not have enough extreme high SBP cases to conduct further subgroup analysis, the positive relationship between SBP and collaterals (represented by decreased $\mathrm{rFTD}$ ) remain in over $170 \mathrm{~mm} \mathrm{Hg}$ range in this study (Figure 3). In other words, we did not find a "ceiling effect" imposed by the blood pressure on collateral status. The potential explanations behind these differences involve different baseline characteristics and collateral scales. Comparing with Rusanen et al.'s2 research which included 104 patients, we had an over doubled sample size with a higher average age (74 yr vs. 68.7 yr), larger proportion of diabetes (26.7\% vs. $16 \%$ ) and higher NIHSS (16 vs. 14) on baseline. On the other hand, unlike our study, Rusanen et al's result based on Souza's ${ }^{25}$ collateral grading scale relying on CTA was limited by the shortage of the imaging technology.

Another unique feature in our study is the grading assessment to measure collaterals. A resent systemic review ${ }^{26}$ com- $^{-}$ pares conventional digital subtraction angiography (DSA), CT, magnetic resonance ${ }^{27-29}$ and transcranial Doppler ${ }^{30}$ and found the latter two techniques are rarely used. digital subtraction angiography as a frequently used grading scale built on the anatomic extent of retrograde flow remains the method to measure collateral extent and number ${ }^{1,31,32}$ however, with risks of complications (e.g. puncture site hematoma, thromboembolic events etc.). ${ }^{33} \mathrm{CT}$ angiography utilizing Thrombolysis In Cerebral Infarction with minor modifications obtains good inter-observer reliability ${ }^{34,35}$ and correlation with clinical outcome, ${ }^{2,7,36}$ however, the result it provided is static (rather than dynamic) with the arterial morphology as a "snapshot" in time instead of dynamic information. In contrast, rFTD method based on CTP-SI in our study can provide a dynamic estimation of leptomeningeal collaterals without the disadvantages of digital subtraction angiography with its inherent invasiveness.

There are several limitations in our study. First, this is a retrospective study in a selected population of ischemic stroke 
patients with pretreatment images and blood pressure records, therefore we have excluded a group of patients due to lack of completed data and cannot perform further comparative study in dynamic blood pressure and reliable collateral circulation changing. Second, the assessment of rFTD is limited to arteries in the Sylvian fissure. On this basis, alternative collateral flow in posterior circulation cannot be assessed and will require other grading methods to visualize the regions such as occipital lobe and cerebellum. Finally, there is a potential increase in Type II error for interaction analysis due to the possible lack of power.

\section{Conclusions}

In conclusion, we showed that in hyperacute phase of ischemic stroke, higher arterial blood pressure is associated with improved leptomeningeal collaterals as exemplified by decrease rFTD. Further studies are needed to confirm the association between $A B P$ and leptomeningeal collaterals and to clarify the effects of hypertensive treatment on clinical outcome.

\section{References}

1. Christoforidis $G A$, Karakasis $C$, Mohammad $Y$, Caragine $L P$, Yang M, Slivka AP. Predictors of hemorrhage following intra-arterial thrombolysis for acute ischemic stroke: the role of pial collateral formation. AJNRAm J Neuroradiol 2009;30:165170.

2. Maas MB, Lev MH, Ay H, Singhal $A B$, Greer DM, Smith WS, et al. Collateral vessels on CT angiography predict outcome in acute ischemic stroke. Stroke 2009;40:3001-3005.

3. Miteff F, Levi CR, Bateman GA, Spratt N, McElduff P, Parsons MW. The independent predictive utility of computed tomography angiographic collateral status in acute ischemic stroke. Brain 2009;132(Pt 8):2231-2238.

4. Menon BK, Smith EE, Modi J, Patel SK, Bhatia R, Watson TW, et al. Regional leptomeningeal score on $\mathrm{CT}$ angiography predicts clinical and imaging outcomes in patients with acute anterior circulation occlusions. AJNR Am J Neuroradiol 2011;32:1640-1645.

5. Bang OY, Saver JL, Kim SJ, Kim GM, Chung CS, Ovbiagele B, et al. Collateral flow predicts response to endovascular therapy for acute ischemic stroke. Stroke 2011;42:693-699.

6. Calleja Al, Cortijo E, García-Bermejo P, Gómez RD, PérezFernández $\mathrm{S}$, Del Monte JM, et al. Collateral circulation on perfusion-computed tomography-source images predicts the response to stroke intravenous thrombolysis. Eur $\mathrm{J}$ Neurol 2013;20:795-802.
7. Lima FO, Furie KL, Silva GS, Lev MH, Camargo ÉC, Singhal AB, et al. The pattern of leptomeningeal collaterals on $\mathrm{CT}$ angiography is a strong predictor of long-term functional outcome in stroke patients with large vessel intracranial occlusion. Stroke 2010;41:2316-2322.

8. Liebeskind DS, Tomsick TA, Foster LD, Yeatts SD, Carrozzella J, Demchuk AM, et al. Collaterals at angiography and outcomes in the interventional management of stroke (IMS) III trial. Stroke 2014;45:759-764.

9. Marks MP, Lansberg MG, Mlynash $M$, Olivot JM, Straka $M$, Kemp $\mathrm{S}$, et al. Effect of collateral blood flow on patients undergoing endovascular therapy for acute ischemic stroke. Stroke 2014;45:1035-1039.

10. Qureshi Al. Acute hypertensive response in patients with stroke: pathophysiology and management. Circulation 2008;118:176-187.

11. Shin HK, Nishimura M, Jones PB, Ay H, Boas DA, Moskowitz $M A$, et al. Mild induced hypertension improves blood flow and oxygen metabolism in transient focal cerebral ischemia. Stroke 2008;39:1548-1555.

12. Hillis $A E$, Ulatowski JA, Barker PB, Torbey M, Ziai W, Beauchamp NJ, et al. A pilot randomized trial of induced blood pressure elevation: effects on function and focal perfusion in acute and subacute stroke. Cerebrovasc Dis 2003;16:236246.

13. Yamauchi H, Higashi T, Kagawa S, Kishibe Y, Takahashi M. Impaired perfusion modifies the relationship between blood pressure and stroke risk in major cerebral artery disease. $J$ Neurol Neurosurg Psychiatry 2013;84:1226-1232.

14. Vemmos KN, Tsivgoulis G, Spengos K, Zakopoulos N, Synetos $A$, Manios $E$, et al. U-shaped relationship between mortality and admission blood pressure in patients with acute stroke. $J$ Internal Med 2004;255:257-265.

15. Castillo J, Leira R, García MM, Serena J, Blanco M, Dávalos A. Blood pressure decrease during the acute phase of ischemic stroke is associated with brain injury and poor stroke outcome. Stroke 2004;35:520-526.

16. Leonardi-Bee J, Bath PM, Phillips SJ, Sandercock PA; IST Collaborative Group. Blood pressure and clinical outcomes in the international stroke trial. Stroke 2002;33:1315-1320.

17. Cao W, Campbell BC, Dong Q, Davis SM, Yan B. Relative filling time delay based on CT perfusion source imaging: a simple method to predict outcome in acute ischemic stroke. AJNR Am J Neuroradiol 2014;35:1683-1687.

18. Campbell BC, Yassi N, Ma H, Sharma G, Salinas S, Churilov L, et al. Imaging selection in ischemic stroke: feasibility of automated CT-perfusion analysis. Int J Stroke 2015;10:51-54.

19. Straka $M$, Albers GW, Bammer R. Real-time diffusion-perfu- 
sion mismatch analysis in acute stroke. J Magn Reson Imaging 2010;32:1024-1037.

20. Cosgrave L, Bernhardt J, Churilov L, Indredavik B, Cumming T. Gender and being born overseas influences the amount of acute stroke therapy. J Rehabil Med 2013;45:130-136.

21. Long JS, Freese J. Regression models for categorical dependent variables using stata. 2nd ed. College Station, TX: Stata Press, 2006.

22. Rusanen H, Saarinen JT, Sillanpää N. The association of blood pressure and collateral circulation in hyperacute ischemic stroke patients treated with intravenous thrombolysis. Cerebrovasc Dis 2015;39:130-137.

23. Kim HJ, Kang DW. Induced hypertensive therapy in an acute ischemic stroke patient with early neurological deterioration. J Clin Neurol 2007;3:187-191.

24. He J, Zhang Y, Xu T, Zhao O, Wang D, Chen CS, et al. Effects of immediate blood pressure reduction on death and major disability in patients with acute ischemic stroke: the CATIS randomized clinical trial. JAMA 2014;311:479-489.

25. Souza LC, Yoo AJ, Chaudhry ZA, Payabvash $S$, Kemmling $A_{t}$ Schaefer PW, et al. Malignant CTA collateral profile is highly specific for large admission DWI infarct core and poor outcome in acute stroke. AJNR Am J Neuroradiol 2012;33:13311336.

26. McVerry F, Liebeskind DS, Muir KW. Systematic review of methods for assessing leptomeningeal collateral flow. AJNR Am J Neuroradiol 2012;33:576-582.

27. Wu B, Wang X, Guo J, Xie S, Wong EC, Zhang J, et al. Collateral circulation imaging: MR perfusion territory arterial spin-labeling at 3T. AJNR Am J Neuroradiol 2008;29:18551860.

28. Chng SM, Petersen ET, Zimine I, Sitoh YY, Lim CT, Golay X. Territorial arterial spin labeling in the assessment of collateral circulation: comparison with digital subtraction angiography. Stroke 2008;39:3248-3254.
29. Chalela JA, Alsop DC, Gonzalez-Atavales JB, Maldjian JA, Kasner SE, Detre JA. Magnetic resonance perfusion imaging in acute ischemic stroke using continuous arterial spin labeling. Stroke 2000;31:680-687.

30. Kim Y, Sin DS, Park HY, Park MS, Cho KH. Relationship between flow diversion on transcranial Doppler sonography and leptomeningeal collateral circulation in patients with middle cerebral artery occlusive disorder. J Neuroimaging 2009;19:23-26.

31. Christoforidis GA, Mohammad Y, Kehagias D, Avutu Bindu, Slivka AP. Angiographic assessment of pial collaterals as a prognostic indicator following intra-arterial thrombolysis for acute ischemic stroke. AJNR Am J Neuroradio/ 2005;26:17891797.

32. Higashida RT, Furlan AJ, Roberts $H$, Tomsick T, Connors B, Barr J, et al. Trial design and reporting standards for intraarterial cerebral thrombolysis for acute ischemic stroke. Stroke 2003;34:e109-e137.

33. Cloft HJ, Joseph GJ, Dion JE. Risk of cerebral angiography in patients with subarachnoid hemorrhage, cerebral aneurysm, and arteriovenous malformation: a meta-analysis. Stroke 1999;30:317-320.

34. Lee KH, Cho SJ, Byun HS, Na DG, Choi NC, Lee SJ, et al. Triphasic perfusion computed tomography in acute middle cerebral artery stroke: a correlation with angiographic findings. Arch Neurol 2000;57:990-999.

35. Kinoshita T, Ogawa T, Kado H, Sasaki N, Okudera T. CT angiography in the evaluation of intracranial occlusive disease with collateral circulation: comparison with MR angiography. Clin Imaging 2005;29:303-306.

36. Tan IY, Demchuk AM, Hopyan J, Zhang L, Gladstone D, Wong $\mathrm{K}$, et al. CT angiography clot burden score and collateral score: correlation with clinical and radiologic outcomes in acute middle cerebral artery infarct. AJNR Am J Neuroradiol 2009;30:525-531. 